\title{
Flow Calculation of Moving Body on Cartesian Grid by Using New Cell-Merging Method
}

\author{
By Paulus Robertus LAHUR and Yoshiaki NAKAMURA \\ Department of Aerospace Engineering, Nagoya University, Nagoya, Japan
}

(Received March 12th, 2001)

\begin{abstract}
Flow simulation around a moving body is a challenging problem, especially when there is more than one body moving relative to each other. To tackle this problem, a Cartesian grid is used in this paper, where its non-body-fitted property allows the grid to stay stationary while the bodies move across it. As a result, it requires only local grid modification in the vicinity of body surface, which may lead to a significant saving of computational cost. On the other hand, the body-fitted grids such as structured and tetrahedral-based unstructured grids move with the body; thus global grid modification is necessary during the movement. In the present study we devised a new implementation procedure for an existing two-dimensional cell-merging method to overcome the problem of conservation regarding gas dynamic properties, a problem caused by body movement across a grid. The present method may have a better potential for extension to 3D. It is based on our previous algorithm developed for a 3D unstructured Cartesian grid and was successfully applied to several test cases in this study. In particular, the efficiency of the method is greatly improved by employing a tree-based data structure to reduce the time to find body panels during computation of the cell's geometrical properties.
\end{abstract}

Key Words: CFD, Cartesian Grid, Moving Body

\section{Introduction}

Research on the use of Cartesian grids to solve fluid dynamic problems has achieved steady advancement in many aspects. The grid has an inherent capability to treat a complicated geometry because of its non-body-fitted property, which is different from other commonly used grids. ${ }^{1-5)} \mathrm{Al}-$ though its applications to the problem of a stationary body have gained popularity, some factors remain to be solved for the problem of a moving body. The Cartesian grid has a unique property; that is, the grid itself remains stationary as a body moves across it. Thus only the local grid portion intersected by the body surface needs to be modified during the course of computation. By contrast, in body-fitted approaches the grid moves with the body, which requires the operation of overall grid modification at each time step. Furthermore, we must compute additional terms produced by the grid movement in the process of flow solving. In this respect, Cartesian approaches have a potential to reduce the computational cost.

At present, body-fitted grids are commonly employed to treat the problem of a moving body in $2 \mathrm{D}$ and $3 \mathrm{D}$. Research to improve the methods employing these grids is being conducted, which are briefly discussed in Ref. 6. First, in tetrahedral-based unstructured approaches, for example, grid movement, smoothing, and remeshing may be computationally demanding, and grid distortion resulting from body movement may cause deterioration in solution accuracy. Second, in overlapping structured and unstructured

(c) 2001 The Japan Society for Aeronautical and Space Sciences grid approaches, much more computation may be required to smooth the intergrid communication when the overlapping regions undergo complicated changes in time. Moreover, the local solution accuracy may be reduced because of large variations in grid size.

On the other hand, Cartesian grid approaches also have several different problems, because of the grid's non-bodyfitted properties. The problem of moving a body in $2 \mathrm{D}$ on a Cartesian grid has been studied by Bayyuk et al. ${ }^{7)}$ and Yang et al. ${ }^{8)}$ Bayyuk et al. proposed and successfully demonstrated solutions to the problems of appearing and disappearing cells resulting from body movement.

The objective of the present research is to devise a Cartesian grid method to treat the moving body problem with a possible extension to 3D. This research is largely based on our previous researches of a steady flow solver on a 3D Cartesian grid where an unstructured approach was employed. $^{9)}$

\section{Computational Scheme}

The flow is obtained by numerically solving the Euler equations in integral form, which can be written as follows:

$$
\frac{\partial}{\partial t} \int \boldsymbol{U} d \Omega+\int \boldsymbol{F} \cdot \hat{n} d S=0
$$

where $\boldsymbol{U}$ is the state vector, $\boldsymbol{F}$ the flux tensor, $\Omega$ the control volume, $\hat{n}$ the normal vector with unit length of control surface, and $S$ the area of control surface.

Then the FVM is applied to discretize a computational domain. Although most cells in the domain keep their geomet- 
rical properties in time, cells near the body surface are subject to change because the body moves across the stationary grid. When this change is taken into account, the discretization of Eq. (1) can be written as Eq. (2), where the conserved variable of $\Omega \boldsymbol{U}$ is employed instead of $\boldsymbol{U}$. That is, mass is used as a variable instead of density.

$$
(\Omega \boldsymbol{U})^{n+1}=(\Omega \boldsymbol{U})^{n}-\Delta t \sum_{k=1}^{m}(\boldsymbol{F} \cdot \hat{n} S)_{k}{ }^{n}
$$

where $\Delta t$ is a time step, and superscripts $n$ and $n+1$ indicate time levels; $m$ is the total number of cell faces in the cell under consideration, $k$ the face number, $\hat{n}$ the unit normal vector to the face, and $S$ the face area. The computational procedure for Eq. (2) is shown in Fig. 1.

A 3-stage Runge-Kutta method is employed to advance the solution to the next time step. The procedures of body movement and grid modification are repeated in each stage. Flux computation at a cell surface is based on Hännel's flux vector splitting ${ }^{10)}$ by using flow solutions on both sides of the surface, which are calculated by extrapolating the solution at each cell's center to the surface center to obtain second-order accuracy in space. The solution gradient is computed by using a least-square method. To retain the upwind property, the contribution of the neighbor sharing the surface is not taken into account. A minmod-type limiter is then applied to the extrapolated values to avoid numerical oscillations.

A maximum allowable time step can be determined from a maximum value of solid body velocity, $\boldsymbol{V}_{\mathrm{s}}$, and fluid flow

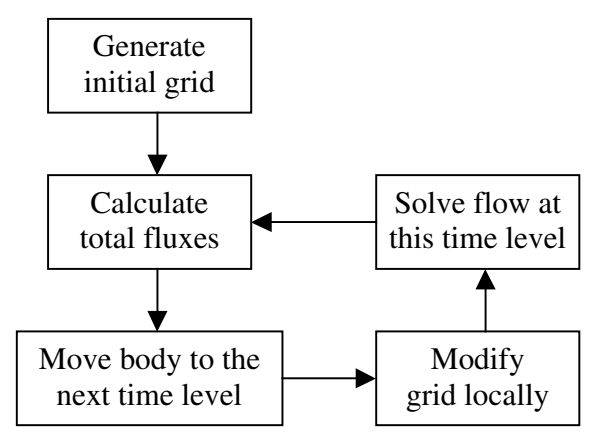

Fig. 1. Flowchart of main computation tasks.

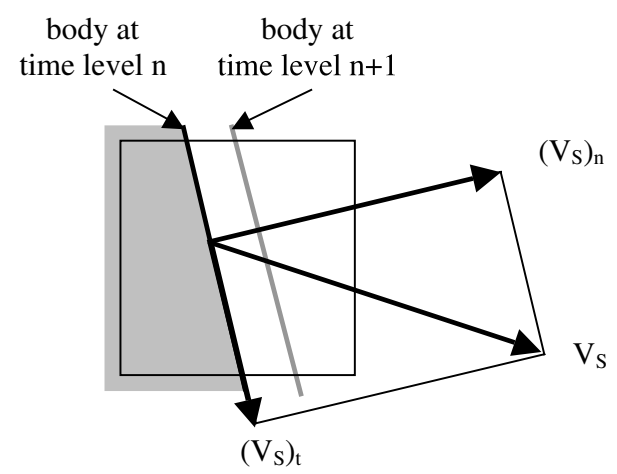

Fig. 2. A cell containing a moving body surface. velocity, $\boldsymbol{V}$, inside all cells, as follows:

$$
\Delta t=\frac{C F L \cdot \Delta L_{\min }}{\max \left(\left|\boldsymbol{V}_{\mathrm{s}}\right|,|\boldsymbol{V}|\right)+C}
$$

where $\Delta L_{\min }$ is the smallest length of fluid cells, or a fraction of it.

Flux computation at an interface in the case of a moving body is different from that in the stationary case because the contribution of energy resulting from the velocity of the body surface and the cell pressure must be considered (see Fig. 2). Assuming these directional senses, as shown in Fig. 2, the normalized flux can be written as follows:

$$
\boldsymbol{F}_{n}=\left(\begin{array}{llllll}
0 & P & 0 & 0 & P\left(V_{\mathrm{s}}\right)_{n}
\end{array}\right)^{\mathrm{T}}
$$

where ()$^{\mathrm{T}}$ denotes a transpose.

\section{The Method of Cut-Cell Merging}

\subsection{Basic principles}

Cartesian grid cells used in the present method may be classified into three cells: (1) a fluid cell, (2) a solid cell, and (3) a cut cell that contains both fluid and solid regions because of intersection with the body surface. Only cells belonging to classes (1) and (3) are used for gas-dynamic computation.

A basic problem in the treatment of a moving body using a Cartesian grid is that a cell may "disappear", or it may become a solid cell because of the movement of a body across a stationary Cartesian grid. In this case, Eq. (2) cannot be used, since the cell has no gas-dynamic properties. Therefore the computed total flux in this cell is not transferred to the next time level; thus the conservation is violated. The opposite situation occurs when a cell inside body "appears", or enters the fluid region outside the body. The cell keeps the gas-dynamic properties at a previous time level, which is physically meaningless.

Modifying the equation to take into account these nonphysical changes in a cell's gas-dynamic properties is too complicated and computationally costly. Instead, Bayyuk et al. suggested a simple alternative, where a cut cell is merged with its suitable neighbor so that the merged cell remains as a cut cell during a one-time step. ${ }^{7)}$ After the step is completed, the cell is separated back to its constituent cells (see Fig. 3). In essence, Eq. (2) is rewritten into a general form of Eq. (5a) for these cells, where A and B denote the constituent cells to be merged.

$$
\begin{aligned}
{\left[(\Omega \boldsymbol{U})_{\mathrm{A}}+(\Omega \boldsymbol{U})_{\mathrm{B}}\right]^{n+1} } & \\
= & {\left[(\Omega \boldsymbol{U})_{\mathrm{A}}+(\Omega \boldsymbol{U})_{\mathrm{B}}\right]^{n}-\Delta t\left\{\left(\sum_{k=1}^{m}(\boldsymbol{F} \cdot \hat{n} S)_{k}\right)_{\mathrm{A}}\right.} \\
& \left.+\left(\sum_{k=1}^{m}(\boldsymbol{F} \cdot \hat{n} S)_{k}\right)_{\mathrm{B}}\right\}^{n}
\end{aligned}
$$

Specifically, if cell A "disappears," its total flux will be transferred to cell B before the time-stepping procedure 
(a)

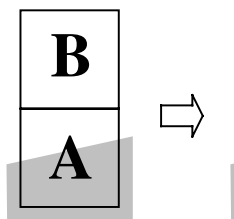

merge cells

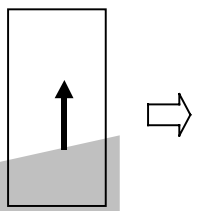
move body
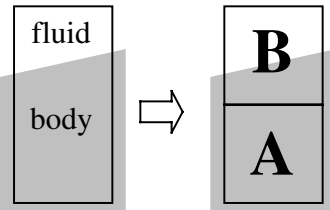
separate cell

(b)
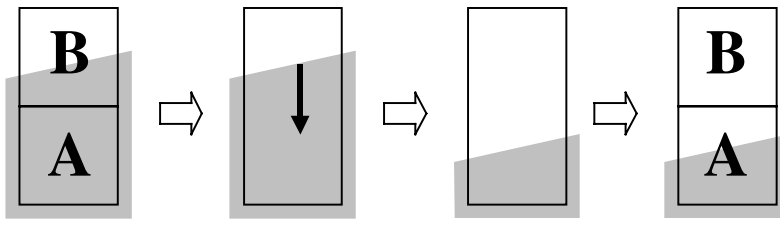

Fig. 3. Cell-merging technique to treat (a) "disappearing" and (b) "appearing" cells.

ends, such as shown in Eq. (5b). On the other hand, if cell A "appears," it will receive part of the total flux from cell B after the time step is completed, as shown in Eq. (5c). Thus the conservation of each physical quantity is always maintained.

$$
\begin{aligned}
& {\left[(\Omega \boldsymbol{U})_{\mathrm{B}}\right]^{n+1}=\left[(\Omega \boldsymbol{U})_{\mathrm{A}}+(\Omega \boldsymbol{U})_{\mathrm{B}}\right]^{n}} \\
& -\Delta t\left\{\left(\sum_{k=1}^{m}(\boldsymbol{F} \cdot \hat{n} S)_{k}\right)_{\mathrm{A}}+\left(\sum_{k=1}^{m}(\boldsymbol{F} \cdot \hat{n} S)_{k}\right)_{\mathrm{B}}\right\}^{n} \\
& {\left[(\Omega \boldsymbol{U})_{\mathrm{A}}+(\Omega \boldsymbol{U})_{\mathrm{B}}\right]^{n+1}} \\
& =\left[(\Omega \boldsymbol{U})_{\mathrm{B}}\right]^{n}-\Delta t\left\{\left(\sum_{k=1}^{m}(\boldsymbol{F} \cdot \hat{n} S)_{k}\right)_{\mathrm{B}}\right\}^{n}
\end{aligned}
$$

To summarize, Eq. (5a) suggests the following computational strategy:

(1) Compute the total flux of all cells at time level $n$;

(2) Merge the cut cells;

(3) Move the body to the next time level, $n+1$;

(4) Compute cell geometrical properties;

(5) Compute the solution;

(6) Separate the merged cut cells.

Cell merging is also a common practice in Cartesian grid approaches to treat very small cut cells, which can greatly reduce the size of a time step. Furthermore, it has been shown by Coirier et al. ${ }^{11)}$ that cell merging affects only the local computational accuracy, not its global value. Thus, based on the discussion mentioned above, the cell-merging approach is adopted in the current study.

\subsection{Implementation of cut cell merging}

Although the idea of cell merging is simple, its implementation is not easy. Bayyuk et al. has demonstrated a way to implement the idea in 2D. ${ }^{7)}$ Even if we are concerned with $2 \mathrm{D}$ problems in the present study, it is desirable to propose a new cell-merging algorithm with possible extension to $3 \mathrm{D}$.

An important issue in this implementation is to keep the algorithm simple. To do this, possible complications can be eliminated by preventing the body from moving too fast, since this can result in a solid cell becoming a fluid cell during a one-time step, and vice versa (Fig. 4). Specifically, it can be achieved by enforcing the CFL condition that limits movement of the body surface relative to a cell, as shown in
(1)
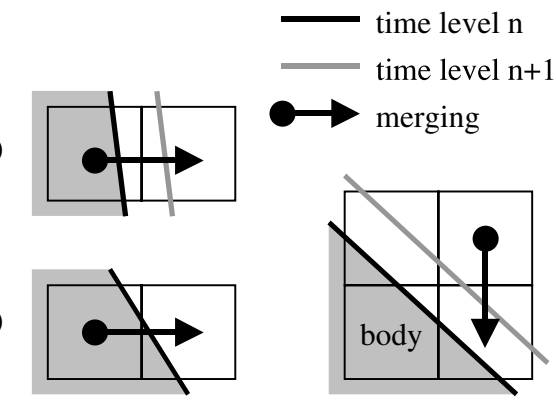

(2)
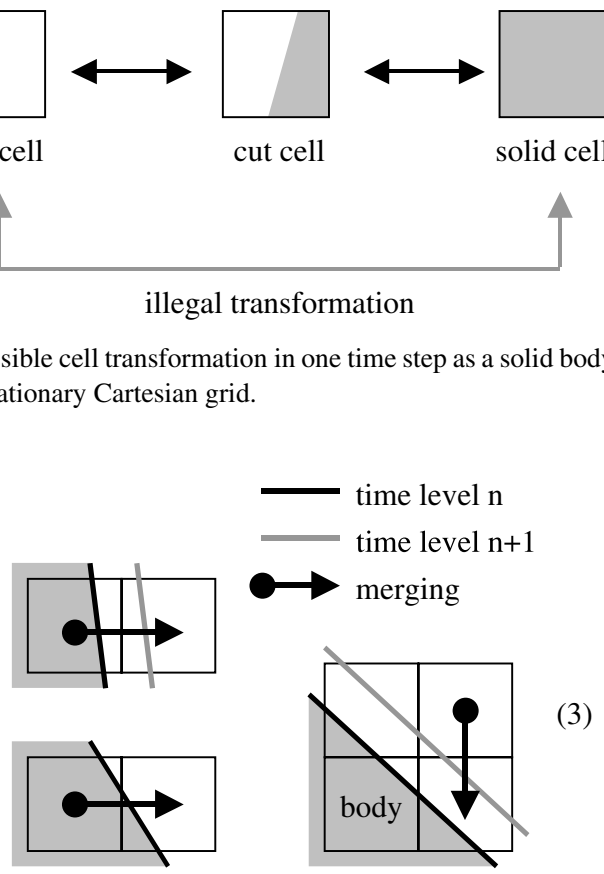

legal transformation

Fig. 4. Possible cell transformation in one time step as a solid body moves across a stationary Cartesian grid.

Fig. 5. Merging steps.

Eq. (3).

The next issue is to determine which cell should be merged and with which cell. This is a very important issue because the effectiveness of the whole method depends on the robustness of this procedure. The procedure proposed in the current research is outlined below.

After identifying cut cells at current and next time levels, the following merging steps are carried out (see. Fig. 5):

(1) Cut cells at time level $n$ are merged with their selected neighbor cut cells at time level $n$ or $n+1$. A valid neighbor is a cell lying in the direction most normal to the body surface inside the cut cell. Cut cells that have no such neighbors are not merged in this step.

(2) All small cut cells at time level $n$ are merged with their neighboring fluid or cut cells at the same time level. Here a small cut cell is defined as a cell with volume less than a threshold value, and the neighbor is defined in the same manner as in step (1).

(3) All cut cells at time level $n+1$ that are not yet merged are merged with any neighbor cut cells at time level $n$.

The only requirement during the merging process is that the basic shape of a Cartesian cell should be preserved. Therefore when a merging group assumes an " $L$ " shape, one or more additional cells must be included in the group to satisfy the requirement. This type of complexity normally occurs in a region where the orientation of body surface is diagonal to the Cartesian grid. In such a situation, the merging group contains typically four cells in $2 \mathrm{D}$. However, there is also a rare occasion when the group includes up to nine cells. An illustration of the cell merging described here is shown in Fig. 6.

The merging of two cells can be carried out by either of 
body surface at time levels:

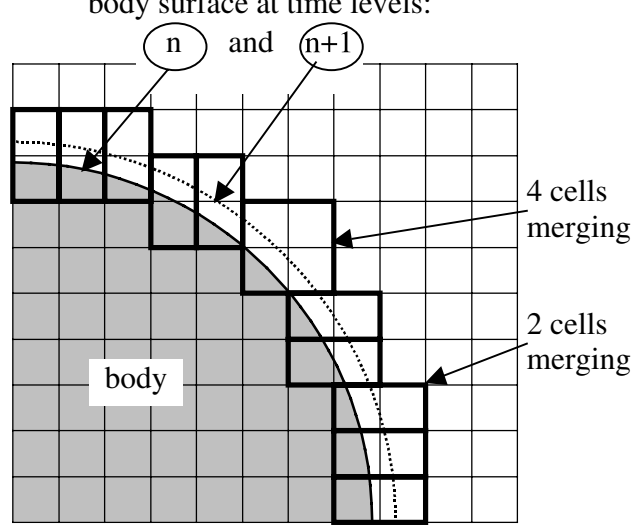

Fig. 6. Cell merging around a body surface.

two methods: (1) removing the physical interface between the cells, or (2) summing the total flux and volume of the cells. As far as the computational effort and results are concerned, there is no strong reason to prefer either of these. Therefore the latter approach is employed here because its implementation is simpler in the present code.

The cell-merging method proposed here has the following advantageous features, compared with the method described in Ref. 7:

1. The process of cell merging can start from any cut cell on the body surface, whereas the method in Ref. 7 requires a suitable starting point.

2. The method in Ref. 7 traverses neighboring cut cells along the body surface in a specified direction, which makes it difficult to extend its implementation to 3D. On the other hand, the present method has no special order, which may lead to an easier extension to 3D.

\section{Grid Generation and Data Structure}

An unstructured approach is employed here, which is an extension of our previous research. ${ }^{9)}$ Thus the connections of cell-to-face and face-to-cell are stored explicitly, which makes finding a neighboring cell very simple and fast. By contrast, a tree-based approach is carried out by traversing up and down the tree, ${ }^{12,13)}$ which requires more computation, though it takes less storage. The advantage of unstructured approaches becomes clear when a solution-based grid adaptation is performed to resolve a flow with anisotropic features. This capability is planned to be incorporated in future research.

The procedure of grid generation is also an extension of our previous research, which is based on 3D. The treatment of a very thin geometry using the cell-splitting algorithm $^{12,13)}$ has not been incorporated into the present code, allowing us to examine the essential parts of the algorithm employed.

An extension of the cell-merging method to include these cases will introduce some degree of complexity. How much complexity will arise depends very strongly on the type of geometry we want to treat. For example, to treat the move- ment of a single clean configuration wing with a thin sharp geometry will require only moderate modification to the existing method. Basically, what the cell-merging algorithm must do is to make sure that after a cell-merging process, a cell with a thin geometry will still contains it at the next time step. This is not difficult to do when the geometry is not too complicated. However, this is not so when the geometry becomes more complicated, such as a thin wing with a moving flap. This is because of the possibility that at some instant a merged cell might contain not only a thin body, but also a thin body with another body surface, or even worse: two thin bodies. The treatment of such geometry will require great effort in its implementation. Avoiding these cases seems to be the best strategy. This can be done by prerefining the grid in the potentially problematic region so that the body surface geometry included within any cell becomes as simple as possible.

One main concern in grid generation and modification is the time taken to perform these tasks, which are largely occupied by computation of the geometrical properties of cut cells. This can be approximated as

$$
T=C N_{\mathrm{C}} f\left(N_{\mathrm{B}}\right)
$$

where $T$ is the total time required to modify the grid, $C$ is some constant, $N_{\mathrm{C}}$ the number of cut cells, and $N_{\mathrm{B}}$ the number of body panels. Without certain precautions, the process may greatly slow down in a situation having many body panels. Thus the following steps have been taken here: (1) Before the computation of the geometrical properties of a cut cell, body panels are checked for possible intersection with the cell; (2) Only body panels near the cell are considered. The first step is necessary because the procedure of cell cutting is computationally much more expensive than the cost of a simple inspection. The second step is to avoid wasting time on examining body panels far from the cell. This reduction in search time is made by employing a tree-based data structure called Alternating Digital Tree (ADT) ${ }^{14)}$ to store body panels. ADT is suitable for storing and searching randomly located objects in space of any dimension. The objects are stored in a "bounding box" before the construction of ADT. In an ideal situation, the search time will become logarithmically proportional to the number of body panels. On the other hand, an alternative method, which is the simplest, is to sweep through the list of body panels. This method will quickly become impractical, however, because the search time is linearly proportional to the number of body panels.

Because of body movement, an ADT can be used only once and must be renewed after the movement. Since constructing ADT takes time, it is desirable to be able to reuse the data. We propose a very simple solution to this problem, that is, expanding the bounding box so that it will contain the body panel even after movement. In the present implementation, ADT is constructed once every time step, which is reused in all three Runge-Kutta stages within the same time step. Note that it is also possible to extend the use of the data to several time steps. 


\section{Test Cases}

\subsection{Moving piston in $1 D$}

This test case is intended to validate the present method by 1D computation adapted from Sod's test problem regarding shock tube. The length of the computational domain is $20 \mathrm{~m}$, which is coarsely discretized into 50 cells in the $X$ direction, with one cell each in the $Y$ and $Z$ directions. The initial stationary fluid inside the tube is divided into two regions in the middle. On the left-hand side, the density is $\rho=1 \mathrm{~kg} / \mathrm{m}^{3}$ and the pressure is $P=100 \mathrm{kPa}$; on the right-hand side, $\rho=0.125 \mathrm{~kg} / \mathrm{m}^{3}$ and $P=10 \mathrm{kPa}$. The resulting distributions of pressure, density, and velocity at $t=0.01 \mathrm{sec}$ are shown in Fig. 7. A comparison with analytical (exact) results shows that the present computational method is capable of capturing flow features such as shock, expansion, and contact discontinuity.
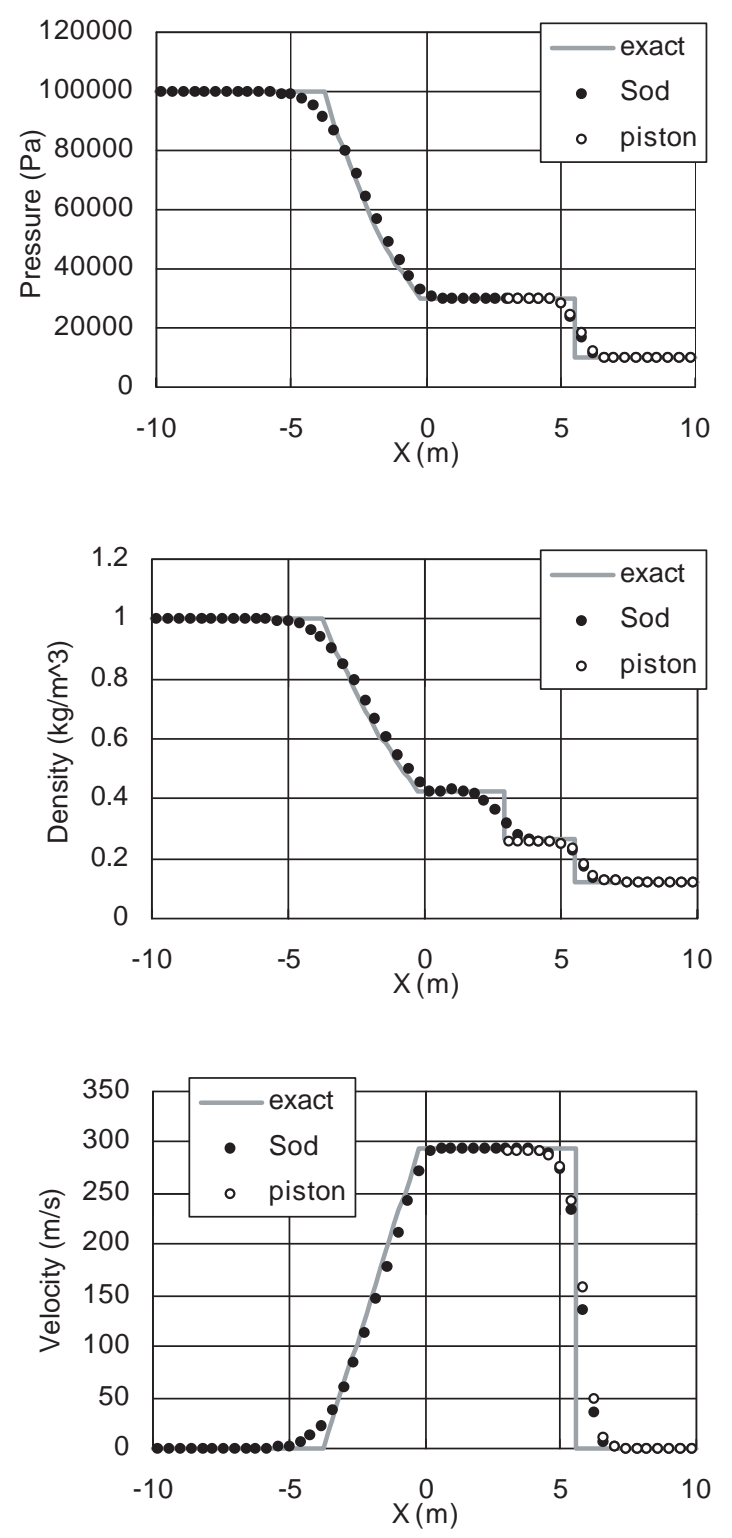

Fig. 7. Comparison between analytical and computational results for a Sod's test problem.
Then for the piston problem, the left half of the fluid is replaced with a solid piston that is forced to move to the right at the same speed $(293 \mathrm{~m} / \mathrm{s})$ as the contact discontinuity in Sod's test problem. It is seen from Fig. 7 that the results are quite close to other results. The only difference is that the contact discontinuity is not smeared in the piston case because it is given as a boundary condition.

This moving piston problem was also computed by using a different frame of reference where an observer is moving with the piston. In this situation, the piston is stationary and the fluid is moving to the left. The results are shown in Fig. 8. Although there is also a slight smearing of shock seen in this case, the overall results are quite close. This confirms that a change in the frame of reference does not significantly affect the flow solution.
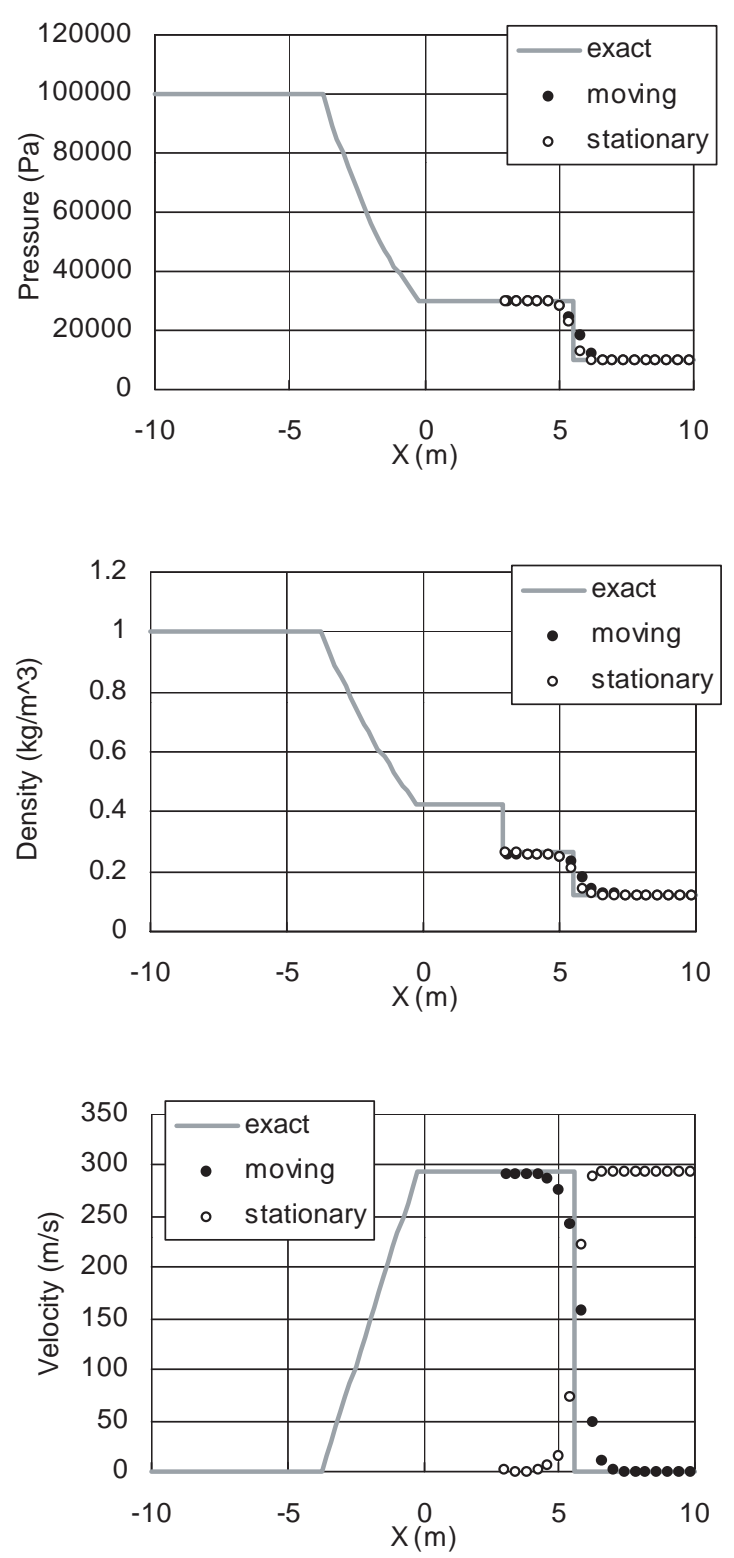

Fig. 8. Comparison between a moving piston in stationary fluid and a stationary piston in moving fluid. 


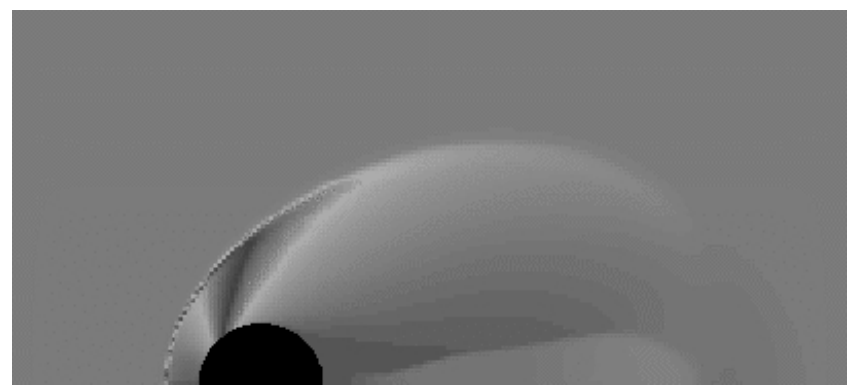

(a)

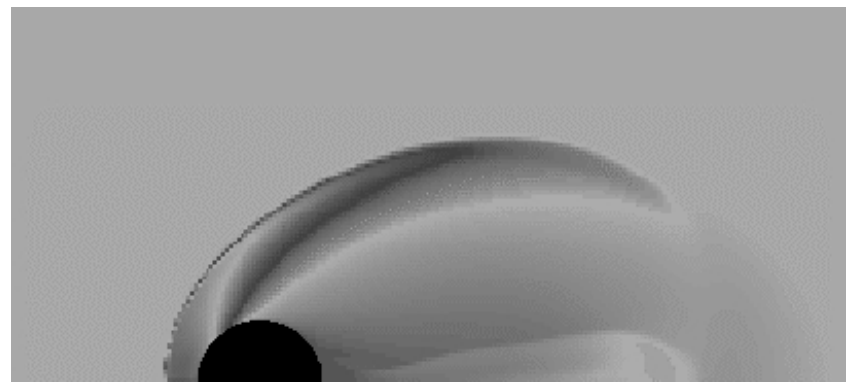

(b)

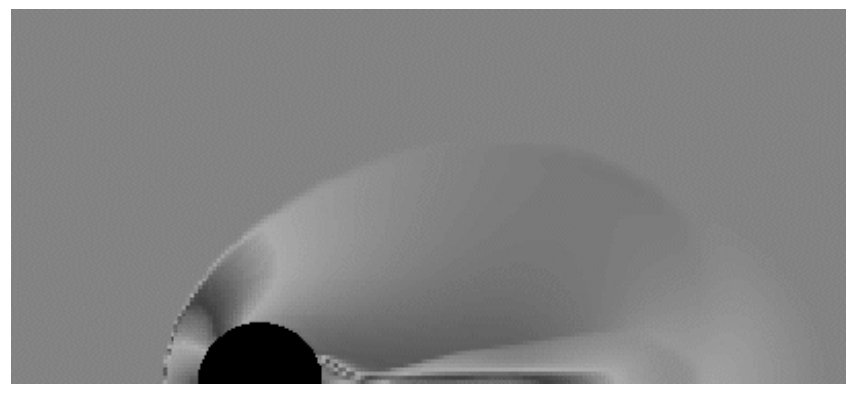

(c)

Fig. 9. Flow moving to the right at $M=3$ around a stationary cylinder (a) pressure, (b) density, and (c) mach number.

\subsection{Moving cylinder in $2 \mathrm{D}$}

The objective of this problem is to validate the present method in the 2D problem by using two different frames of reference: (1) a stationary cylinder in moving fluid, and (2) a moving cylinder in stationary fluid. The relative velocity between the fluid and the cylinder is $M=3$, and a uniform Cartesian grid is employed. To simulate the $2 \mathrm{D}$ flow, only one cell in the $z$ direction is employed under symmetrical boundary conditions. The cylinder diameter is 32 times as large as the side of a cell.

The distributions of pressure, density, and velocity at $t=$ 2 are shown in Figs. 9 and 10, where the results are quite close in both. Note that the magnitude of velocity vectors is different in these two frames of reference. This test case proves that a change in the frame of reference produces no appreciable differences in 2D computation.

\subsection{Multiple bodies in motion}

One objective of this test case is to examine the difference in treatment between a single body and multiple bodies. The other is to study the speed of computation and the factors

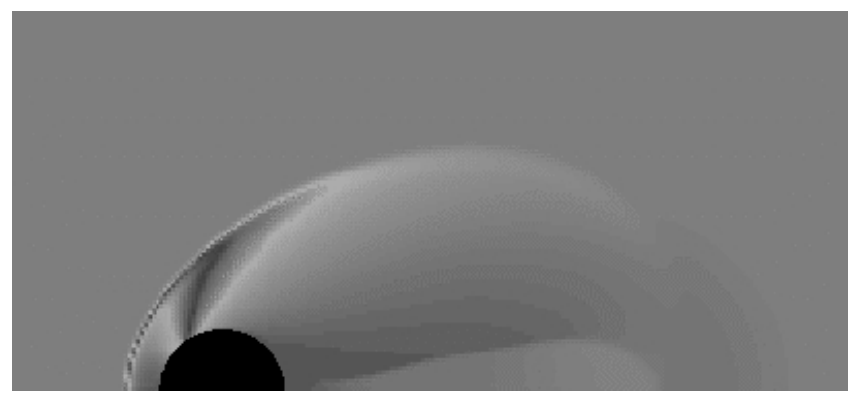

(a)

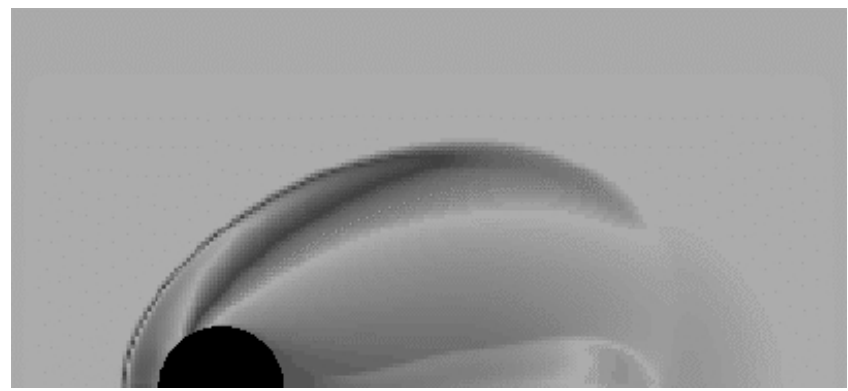

(b)

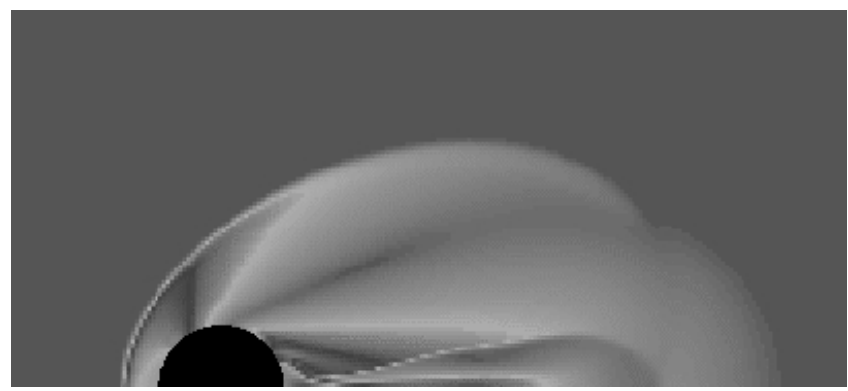

(c)

Fig. 10. Flow induced by cylinder moving to the left at $M=3$ in stationary fluid: (a) pressure, (b) density, and (c) mach number.

that affect it.

Two cylinders with a capsulelike cross-section are considered, which pass each other in a 2D tunnel. The initial locations of the bodies are shown in Fig. 11, along with the grid. The dimension of the tunnel is $384 \times 112 \times 1$ in the $x, y$, and $z$ directions, respectively, where one unit length represents the side of a cubic cell. As in the previous cases, only one cell in the $z$ direction is used to simulate the 2D flow, where the total number of cells is 43,008 . The length and height of each body are 96 and 32 units length, respectively. The bodies move with an impulsive start from rest to a constant velocity of $M=2.0$ in the horizontal, but opposing directions, where the fluid is initially at rest.

The treatment of multiple bodies differs from that of single body in the computation of velocity and the coordinates of the body surface. Furthermore, the bodies should not overlap one another, which would violate a geometrical constraint. The other aspects of flow computation and grid generation are the same as with the single body.

The flow solutions at three instances are shown in Fig. 12. 


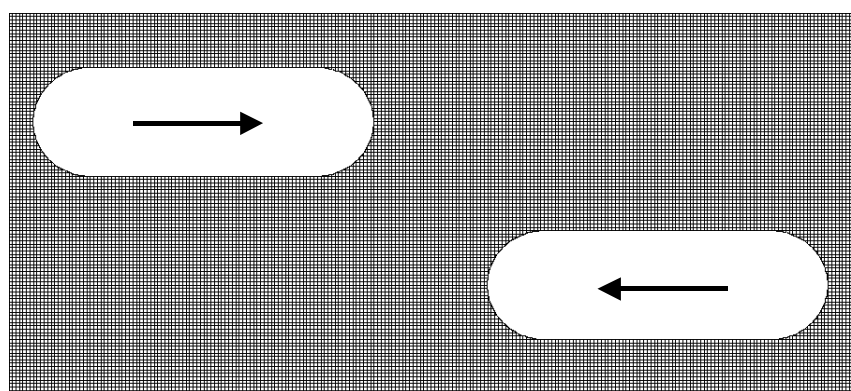

Fig. 11. Grid around 2D capsules at an initial position in a tunnel.

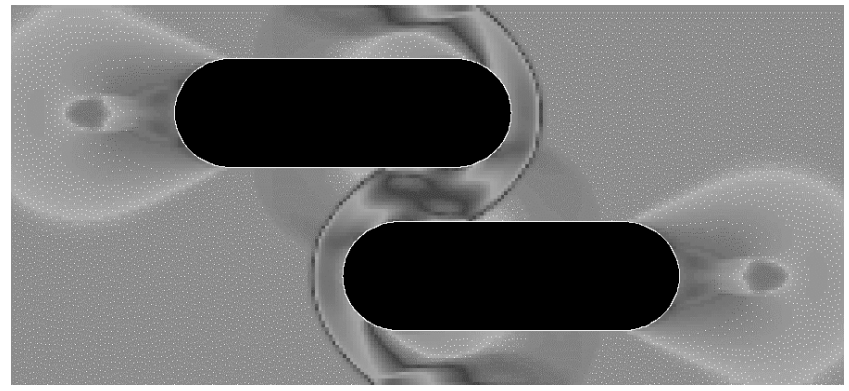

(a)
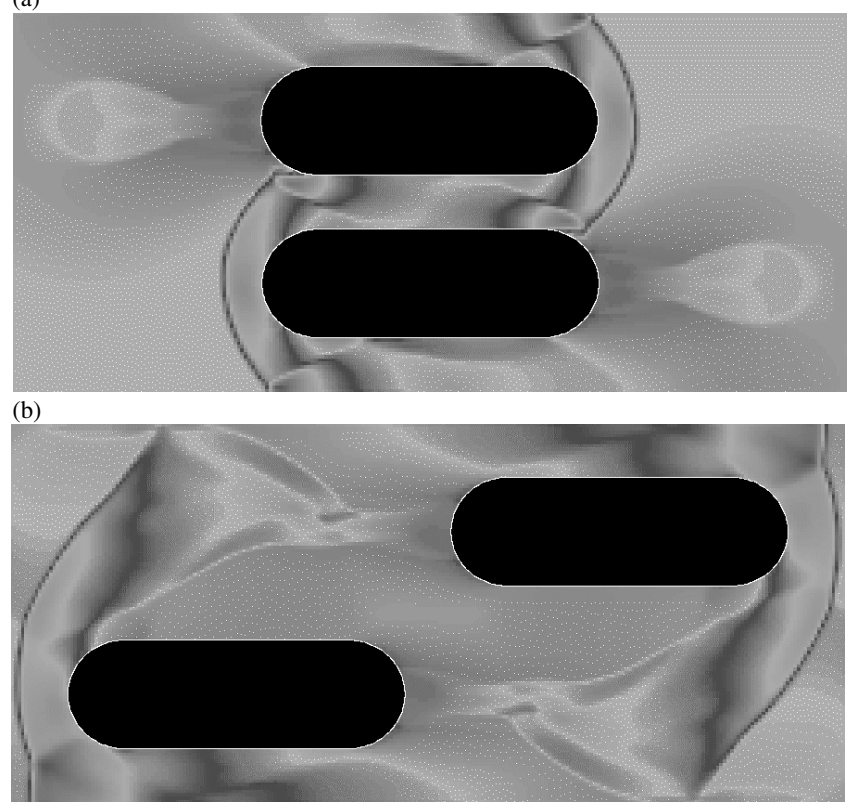

(c)

Fig. 12. Density distribution around 2D capsules moving in opposite directions in a tunnel at three instances: (a) 1.1, (b) 2.0, and (c) 3.7 time units.

The impulsive start creates strong expansion waves behind the bodies. The shocks in front of each body are reflected at the tunnel walls, where the phenomenon of Mach reflection is observed to grow with time. The shocks also hit the solid surface of the other body, and afterward expansion waves generated from the trail of the opposing body interact with the bow shocks.

The initial grid generation takes $1.19 \mathrm{sec}$ of CPU time on a $21164 \mathrm{~A} 600 \mathrm{MHz}$ Alpha workstation. One iteration takes $6.92 \mathrm{sec}$, where flux computation and grid modification take 5.92 and $0.58 \mathrm{sec}$, respectively, i.e., $86 \%$ and $8 \%$ in percentage, respectively. Therefore, the time taken to compute flux per iteration per cell becomes $0.16 \mathrm{~ms}$. Note that the present grid modification time includes three times of modification as a result of the third-order Runge-Kutta scheme. Because cut cells total 512 , the time taken to modify one becomes $0.37 \mathrm{~ms}$.

To study the time taken by grid generation and its modification as a function of the number of body panels, the time measurement was repeated by changing the number of bodies. Each body consists of 328 panels and 166 nodes. The breakdown of time taken for one iteration is presented in Fig. 13, where it is evident that the grid modification time tends to increase with the number of body panels. The reduction in flux computation time was caused by the decrease in the number of fluid cells in the computational domain.

The total time to modify a grid in one iteration is less than that for the initial grid generation, as can be seen in Fig. 14. To examine the advantage of using ADT as a body panel searching algorithm, computations were carried out with and without ADT. In the latter case, a linear search method was employed, where the computation greatly slows down as the number of body panels increases, as shown in Fig. 14.

A more specific measure is the time taken to modify a cut

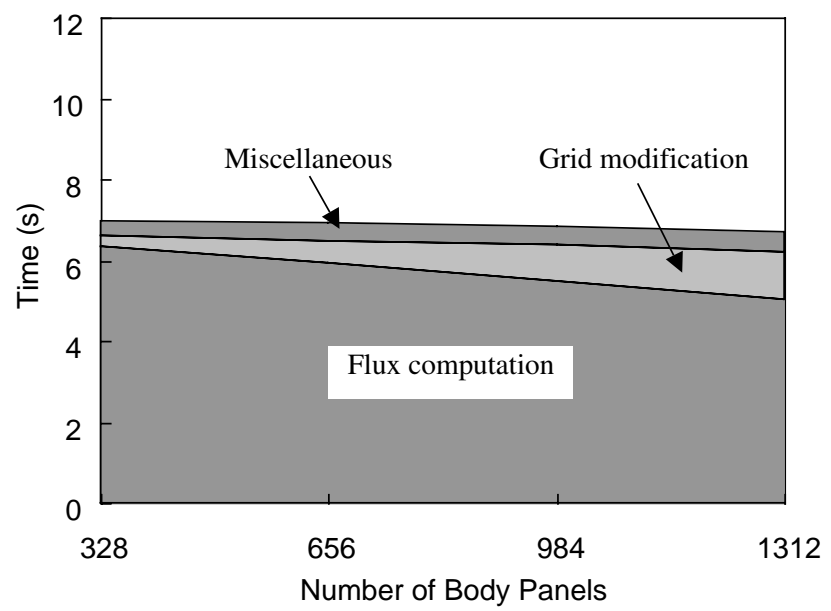

Fig. 13. Breakdown of time to complete one iteration as a function of the number of body panels.

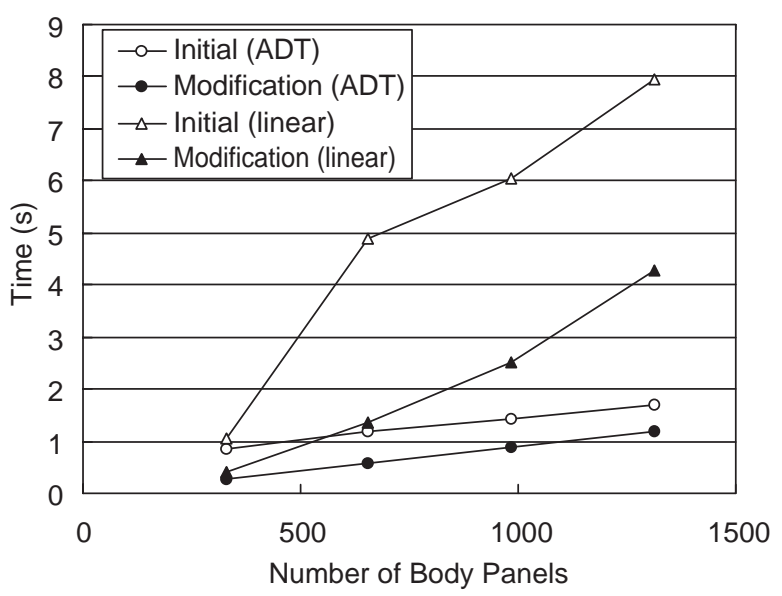

Fig. 14. Grid generation time vs. number of body panels. 


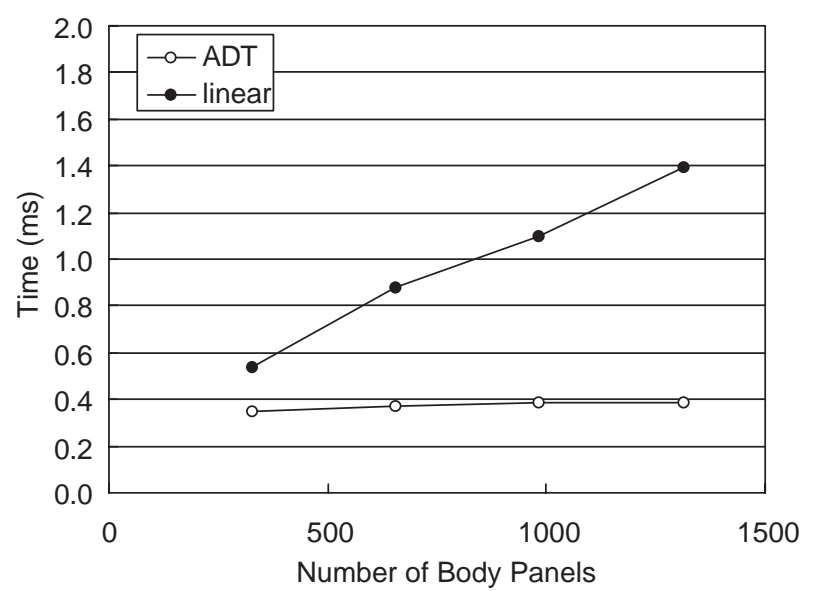

Fig. 15. Time to modify a cut cell.

cell per iteration (Fig. 15). Here it is evident that the time increases more or less logarithmically with the number of body panels when ADT is employed. On the other hand, in linear search the search time increases about linearly with the number of body panels. From these results, the use of ADT is recommended for a great many body panels.

\section{Concluding Remarks}

A new cell-merging method to treat a moving body on a Cartesian grid has been proposed. Unlike the existing method, it does not require (1) searching for a suitable starting point, or (2) traversing along the body surface to proceed. These features are considered to be important in extending the present treatment to $3 \mathrm{D}$ problems.

The results obtained here are summarized as follows:

1. The effectiveness of the present method has been validated for three test cases: a moving piston, a cylinder, and multiple bodies. A change in the reference frame from stationary to moving does not seem to significantly affect the computational results.

2. The efficiency of grid generation and of modification has been largely improved by the use of a tree-based data structure known as ADT, where the time to search for body panels in the procedure of cell cutting has been significantly reduced.
3. The method retains the easy-to-use property of a Cartesian grid, whether the body is single or multiple. Furthermore, because only local grid modification is needed, the present method has the potential to efficiently treat a moving body on a Cartesian grid.

\section{References}

1) Aftosmis, M. J.: Solution Adaptive Cartesian Grid Methods for Aerodynamic Flows with Complex Geometries, Computational Fluid Dynamics VKI Lecture Series 1997-05 (1997).

2) Domel, N. D. and Karman, S. L.: Splitflow: Progress in 3D CFD with Cartesian Omni-tree Grids for Complex Geometries, AIAA Paper 2000-1006 (2000).

3) Wang, Z. J., Chen, R. F., Hariharan, N., Przekwas, A. J. and Grove, D.: A $2^{\mathrm{N}}$ Tree Based Automated Viscous Cartesian Grid Methodology for Feature Capturing, AIAA paper 99-3300 (1999).

4) Pember, R. B., Bell, J. B., Colella, P., Crutchfield, W. Y. and Welcome, M. L.: An Adaptive Cartesian Grid Method for Unsteady Compressible Flow in Irregular Regions, J. Comp. Physics, 120 (1995), pp. 278304.

5) Welterlen, T. J.: Store Release Simulation on the F/A-18C Using Splitflow, AIAA Paper 99-0124 (1999).

6) Löhner, R., Sharov, D., Luo, H. and Ramamurti, R.: Overlapping Unstructured Grids, AIAA Paper 01-0439 (2001).

7) Bayyuk, S. A., Powell, K. G. and van Leer, B.: A Simulation Technique for 2-D Unsteady Inviscid Flows around Arbitrarily Moving and Deforming Bodies of Arbitrary Geometry, AIAA Paper 93-2291-CP (1993).

8) Yang, G., Causon, D. M. and Ingram, D. M.: Cartesian Cut-Cell Method for Axisymmetric Separating Body Flows, AIAA J., 37 (1999), pp. 905-911.

9) Lahur, P. R. and Nakamura, Y.: Anisotropic Cartesian Grid Generation, AIAA Paper 2000-2243 (2000).

10) Hännel, D., Schwane, R. and Seider, G.: On the Accuracy of Upwind Schemes for the Solution of the Navier-Stokes Equations, AIAA 871105, Proc. of AIAA 8th Computational Fluid Dynamics Conference (1987), pp. 42-46.

11) Coirier, W. J. and Powell, K. G.: An Accuracy Assessment of Cartesian-Mesh Approaches for The Euler Equations, J. Comput. Physics, 117 (1995), pp. 121-131.

12) Lahur, P. R. and Nakamura, Y.: A Cartesian Grid Generation Method Considering a Complicated Cell Geometry at the Body Surface, Trans. Japan Soc. Aero. Space Sci., 43 (2000), pp. 8-15.

13) Lahur, P. R. and Nakamura, Y.: A New Method for Thin Body Problem in Cartesian Grid Generation, AIAA Paper 99-0919 (1999).

14) Bonet, J. and Peraire, J.: An Alternating Digital Tree (ADT) Algorithm for 3D Geometric Searching and Intersection Problems, Int. J. Num. Methods Eng., 31 (1991), pp. 1-17. 